\title{
Erratum: Identification of three-qubit entanglement [Phys. Rev. A 87, 012316 (2013)]
}

\author{
Ming-Jing Zhao, Ting-Gui Zhang, Xianqing Li-Jost, and Shao-Ming Fei
}

(Received 14 October 2018; published 29 November 2018)

DOI: 10.1103/PhysRevA.98.059901

We would like to correct some inaccuracies and omissions in our article.

(1) The states $\sigma_{b}$ used in example 2 and $\rho_{3}$ in example 3 should not be called positive partial transposition (PPT) states since they are, in fact, not positive under partial transposition with respect to the first and the third qubits, although they can still serve as examples for the application of our separability criterion. Therefore we cannot assert that the inequality (5) has advantages in PPT entanglement detection.

(2) In connection with Eq. (6) and example 2, we accidentally omitted a reference to the work of P. Horodecki [1] where this expression was first presented [see Eq. (32) in that paper].

We acknowledge Dr. A. Singh and Dr. A. Gautam for pointing out these issues.

[1] P. Horodecki, Phys. Lett. A 232, 333 (1997). 